\title{
Pre-eclampsia research in the Norwegian Mother and Child Cohort Study
}

\author{
Per Magnus and Lill Trogstad \\ Norwegian Institute of Public Health, Oslo, Norway \\ Correspondence: Per Magnus, Norwegian Institute of Public Health, P.O. Box 4404 Nydalen, NO-0403 Oslo, Norway \\ e-mail: per.magnus@fhi.no Telephone: +4721078211
}

\begin{abstract}
Pre-eclampsia is a pregnancy disorder of unknown origin. In Norway, pre-eclampsia is reported in 3 to 4 per cent of registered births. At present, November 2014, a series of investigations into the etiology of preeclampsia have been published internationally from the Norwegian Mother and Child Cohort Study (MoBa), and several studies are ongoing. The intention of this paper is to give a short summary of what has been accomplished, and to discuss future avenues of research concerning causes, mechanisms and consequences of pre-eclampsia. The papers that have been published up to now include seven that concern life-styles (physical activity, tobacco and diet), six that include prior pregnancies, infection, gestational weight gain, toxicants and tryptophan metabolism, and two studies concerning issues of selection and validity. Major findings are that tobacco smoking is only associated with reduced risk of pre-eclampsia when it occurs in the last trimester; that processed food and sugar-sweetened beverages are associated with increased risk while vegetables, vitamin D and probiotics are associated with reduced risk; and that prior induced abortions have the same risk-reducing effect as a prior pregnancy. For future studies, we suggest that better use should be made of the family structure built into MoBa. This includes better use of the discordant pregnancy design. A series of ongoing genetic studies, partly in international consortia, will hopefully open new etiological insights. The indications that pre-eclampsia is related to cardiovascular disease and other complex disorders should be further investigated through systematic follow-up of pre-eclamptic women and controls. Finally, $\mathrm{MoBa}$ is eminently suited to study the influences that pre-eclampsia can have on the growing child.
\end{abstract}

This is an open access article distributed under the Creative Commons Attribution Licence, which permits unrestricted use, distribution, and reproduction in any medium, provided the original work is properly cited.

\section{INTRODUCTION}

Pre-eclampsia is an ill-defined disease with unknown etiology that each year causes a large number of maternal and fetal deaths worldwide, as well as long-term disabilities in children born prematurely [1]. In societies without sufficient antenatal care, the disease is sometimes not recognized until the occurrence of the dramatic convulsions of eclampsia. Eclampsia is a lifethreatening condition characterized by cerebral edema and liver failure. Fortunately, in societies with sufficient antenatal care, blood pressure is taken and any presence of elevated levels of protein in the urine is recorded at regular visits, which means that early signs of disease can be detected and sometimes treated. The only real cure is the delivery of the placenta, but other remedies have been recommended to reduce symptoms such as antihypertensive medication and tranquilizers. Many randomized, controlled trials have been performed without much success. At present, aspirin is recommended and believed to act through antiinflammatory mechanisms [2].

The reason that pre-eclampsia can be characterized as ill-defined, lies in the fact that the commonly employed criteria for setting the diagnosis are elevated blood pressure after week 20 combined with proteinuria. But both blood pressure and protein levels fluctuate, and it is probable that many cases, especially those occurring in the last part of pregnancy, go undetected, since hypertension and proteinuria may not be present at the same antenatal visit. Some investigators have proposed that pre-eclampsia is only the tip of the iceberg, i.e. that pregnancy represents a heightened state of inflammation that can vary from person to person [3]. For each woman, the presence of overt disease will depend on the length of gestation as well as characteristics such as body mass index (BMI), parity, prior pregnancy outcomes, diabetes and multifetal pregnancy, to name a few that are considered wellestablished risk factors for pre-eclampsia. The heterogeneous nature of the disorder has led to some pessimism as to the possibility to find etiological factors with large effects.

Genetic factors have long been suspected of being responsible for at least part of the etiology, based on familial aggregation and twin studies. To what extent maternal, fetal or maternal-fetal genetic interaction is responsible is not clear. As yet, no genes have been replicated with sufficient statistical significance. A large number of biomarkers have been associated with pre-eclampsia, but there is not yet any established predictive model based on data from the first half of pregnancy that can be used clinically with any great precision. Some of these associated biomarkers may point us towards genes or environmental exposures of etiological significance, but many of them are presumably only downstream consequences of the many metabolic alterations induced by pre-eclampsia. 
The intention of this paper is to give an overview of the scientific activity in the Norwegian Mother and Child Cohort Study (MoBa) as it relates to investigations of pre-eclampsia, and to discuss future avenues of research. MoBa is a large pregnancy cohort with a built-in family design. The cohort is described in detail in preceding papers in this issue, and information is also available on www.fhi.no/moba.

\section{MoBA PUbLiCATIONS}

\section{Smoking}

One of the enigmas in pre-eclampsia research has been the negative association to smoking. It has repeatedly been found that women who smoke have a lower risk of developing the disease. In order to understand the biological mechanisms behind this observation, Stephanie M. Engel from the University of North Carolina received a grant from the National Institutes of Health in the US. The aim has been to use DNA from case and control dyads (mother and child) in $\mathrm{MoBa}$ to genotype polymorphic genetic markers (single nucleotide polymorphisms - SNPs) in metabolic pathways in which tobacco might have an influence. The aims have now been extended to include the complete genome, in a GWAS study. If the genetic analyses can point to new genes that reveal important pathophysiological mechanisms, it may lead to the development of better therapies. As part of this project, an association study between smoking and pre-eclampsia was performed. Exposure to tobacco was based on responses to two questionnaires, sent to the pregnant women in gestational weeks 15 to 17 and week 30, while the information on pre-eclampsia was derived from the birth record in the Medical Birth Registry of Norway. It was found that, whereas $23 \%$ of the pregnant women reported smoking in the first trimester, only 9 and $8 \%$ smoked in the second and third trimester, respectively [4]. The main finding was that the risk of pre-eclampsia was only reduced for women who continued to smoke in the third trimester (adjusted OR $=0.57,95 \% \mathrm{CI}$ : 0.46 0.70 ). Women who quit smoking before the last trimester had about the same risk as non-smokers. No effect of passive smoking could be found. Thus, this observation confirms earlier reports of the apparent protective effect of smoking, but limits the effect to smoking in the last trimester.

\section{Nutrition}

There is a large interest in the effects of nutritional factors on the risk of pre-eclampsia. In MoBa, a semiquantitative food frequency questionnaire has been developed [5]. An impressive number of publications, more than 50, have been published up to now, based on dietary data. In an accompanying paper, Brantsæter et al. gives a summary of the methodology and the results of the investigations, including a discussion of the strengths and limitations of nutritional studies during pregnancy [6].
A previous study that reported reduced levels in early pregnancy of serum 25-hydroxyvitamin D for women who later developed pre-eclampsia [7], prompted a study of the relationship between vitamin D supplementation and pre-eclampsia. It was found that the relative risk of pre-eclampsia for women with a total vitamin D intake of 15-20 grams per day compared to less than 5 grams per day was 0.76 (95\% CI: $0.60-$ 0.95 ) [8]. No effect of dietary vitamin $\mathrm{D}$ alone was found. This protective effect is in line with other reports, although it has recently been concluded that the quality of evidence is insufficient to determine a causal association between vitamin $\mathrm{D}$ and pre-eclampsia [9].

Another fascinating observation in $\mathrm{MoBa}$, as regards nutrition and pre-eclampsia, is the finding that intake of probiotic foods appears to reduce the risk of preeclampsia. Probiotics are defined as live microorganisms, which, when administered in adequate amounts, confer a health benefit on the host [10]. Recent years have witnessed an increasing interest in the effects that the bacterial composition in the intestines may have on diseases where inflammation and immunological responses are important. Pregnancy is a condition where many immunological functions undergo changes, and pre-eclampsia is considered to be a state of increased inflammation. The association between intake of probiotics and pre-eclampsia in primiparous women was examined, and it was found that daily intake appeared to reduce the risk $(\mathrm{OR}=0.80,95 \% \mathrm{CI}$ : $0.66-0.96)$ [11].

Intake of dietary sugar has previously been found to increase the risk of pre-eclampsia in Norway [12]. Using MoBa data, it was found that added sugar in the diet was not associated with pre-eclampsia [13]. However, intake of more than $125 \mathrm{ml}$ of sugar-sweetened beverages per day compared to no intake, was associated with increased pre-eclampsia risk $(\mathrm{OR}=1.27,95 \%$ CI: 1.05-1.27). In the discussion, the authors speculate that the association to sweet drinks is unlikely to be due to the added sugar. They also report that higher intakes of fresh and dried fruits were associated with reduced risk of pre-eclampsia.

Instead of studying effects of single nutrients or foods, the questionnaire also opens for the study of effects of dietary patters. Using principal component analysis, Brantsæter et al (14) showed that four dietary patterns emerged with sufficiently high eigenvalues. These patterns were given the following names based on loadings to certain foods: Vegetable, processed food, potato and fish, and cakes and sweets. Subjects with high factor scores on vegetables had lowest risk of pre-eclampsia, while having high factor scores on processed food was associated with increased risk. Variability in factor scores for potato and fish and cakes and sweets had little influence on the occurrence of pre-eclampsia.

Health effects of organic foods have been little studied, and particularly not in relation to pre-eclampsia. Torjusen and coworkers have recently compared intake 
of organic foods among women in MoBa with and without pre-eclampsia [15]. The food frequency questionnaire included 6 questions on the use of organic foods. No association to pre-eclampsia was found for the consumption of organic fruit, cereals, milk/dairy products, eggs or meat, while higher intake of organic vegetables appeared to decrease the risk of preeclampsia. Comparing subjects who said they consumed organic vegetables often or mostly to subjects saying rarely/never or sometimes gives an OR of 0.75 (95\% CI: 0.60-0.95). The risk reduction associated with use of organic vegetables was observed in addition to the risk reduction associated with healthy diet including vegetables.

Recently, the concept of the New Nordic Diet (NND) has been put forward. A scoring system has been constructed for ten subscales, including such items as meal frequency, Nordic fruits, root vegetables, potatoes, cabbages, whole grain bread, and the like. For the purpose of studying pre-eclampsia, subjects were categorized into low, medium and high NND adherence [16]. When subjects with high adherence to NND were compared to subjects with low adherence, the OR for pre-eclampsia was 0.86 (95\% CI: $0.78-0.95)$. In contrast to the other papers on nutrition, which only studied nulliparous women, this analysis included all women.

\section{Physical activity}

On the basis of analogies and associations between pre-eclampsia and cardiovascular disease, it has been suggested that regular physical activity during pregnancy can reduce the risk of pre-eclampsia. The first $\mathrm{MoBa}$ questionnaire asked how often the women performed 14 different leisure time physical activities, ranging from brisk walking to high-impact aerobic classes. A dose-response relationship was found, with a reduction in the occurrence of pre-eclampsia as the level of physical activity increased (17). For subjects with more than 25 activities per week the relative risk compared to subjects with no activities was $\mathrm{OR}=0.79$ (95\% CI: 0.65-0.96). However, no benefit was found for women with pre-pregnancy body mass index (BMI) above 30.

\section{Pre-pregnancy BMI and weight gain in pregnancy}

Pre-pregnancy BMI above 30 is a well-known risk factor for the development of pre-eclampsia. The effect of a high increase in body weight during pregnancy is less well established. The US Institute of Medicine (IOM) and National Research Council has issued recommendations for gestational weight gain (GWG). In Norway, these guidelines have been adopted and the general advice is that normal weight women are recommended to increase $11.5-16 \mathrm{~kg}$, while the advice for obese women is $5-9 \mathrm{~kg}$. Haugen et al [18] set out to examine the risk of pre-eclampsia and other adverse pregnancy outcomes for women who fell outside these limits. For nulliparous women, the odds ratio for pre- eclampsia was 2.44 (95\% CI: 2.03-2.92) for women who had gained more weight than recommended. For parous women, the corresponding odds ratio was 2.19 (95\% CI: 1.62-2.96). When weight gain in pregnancy is associated with an outcome such as pre-eclampsia, reverse causation must be considered, since edema, a common consequence of pre-eclampsia, will cause increase in weight.

\section{Previous abortions and infertility}

The risk of pre-eclampsia is highest for women delivering their first child. The causes and mechanisms behind this parity effect are not understood. We asked whether a first pregnancy that ended with abortion would lower the risk of pre-eclampsia in subsequent pregnancies. We found that having had two or more previous induced abortions had a strong effect on the later risk of pre-eclampsia, $\mathrm{OR}=0.36(95 \% \mathrm{CI}$ : 0.18 $0.73)$ [19]. One induced abortion had a smaller effect $(\mathrm{OR}=0.87,0.71-1.06)$. The effect after two or more abortions is similar to what is seen after one birth. In contrast, having had one or more miscarriages did not influence the risk of pre-eclampsia in a subsequent pregnancy. Infertility, recurrent miscarriages and preeclampsia may share elements of the same etiological factors. When we looked at recurrent miscarriages (defined as three or more), an increased risk of preeclampsia was found, although not statistically significant $(\mathrm{OR}=1.51,95 \%$ CI: 0.80-2.83) [20]. Furthermore, having experienced infertility (defined as having received a treatment such as in vitro fertilization, operative or hormonal treatment), also had a certain risk-increasing influence $(\mathrm{OR}=1.29,95 \% \mathrm{CI}$ : 1.05 1.60). The combination of recurrent miscarriage and infertility treatment had a stronger effect on the risk of pre-eclampsia $(\mathrm{OR}=2.40,95 \% \mathrm{CI}: 1.11-5.18)$. This finding is in agreement with a genetic theory, proposed by Ashley Moffett from Cambridge University, suggesting that a certain combination of HLA-C alleles in the fetus interacts with haplotypes in the KIR gene in the mother. This can lead to reduced invasion of extravillous trophoblast cells in the decidua, and thereby cause infertility, spontaneous abortions, fetal growth restriction or pre-eclampsia [21]. The genetic details are elaborated in an accompanying paper in this issue [22].

\section{Tryptophan metabolism}

Tryptophan is an essential amino acid. It is a precursor for serotonin. Tryptophan's catabolic route is the kynurenine pathway. The activity in this pathway is influenced by immune responses and inflammatory mediators. Since inflammation is a characteristic of pre-eclampsia, it is of interest to compare the concentrations in plasma of metabolites in this pathway for women with and without pre-eclampsia. In a random subsample of $3000 \mathrm{MoBa}$ participants, who gave birth in the period 2002-2003, the concentrations of tryptophan and six kynurenine pathway metabolites were 
successfully measured in plasma samples, taken at week 17, for 2,936 women. 116 women developed pre-eclampsia. They had increased plasma levels of kynurenine acid (23.3 versus $20.7 \mathrm{nmol} / \mathrm{l})$ [23]. An interaction with BMI was found. In women with BMI above 25 , roughly $20 \%$ of women with level of kynurenine acid above the $95^{\text {th }}$ percentile developed pre-eclampsia, compared to about $4 \%$ for women with BMI above 25 who had kynurenine levels in a reference category (between the $25^{\text {th }}$ and $75^{\text {th }}$ percentile). This result can aid in understanding the pathophysiological processes in the development of pre-eclampsia, but it is not precise enough to be used as a predictive biomarker.

\section{Perfluoroalkyl substances}

Perfluoroalkyl substances (PFAS) are persistent environmental contaminants, used in many consumer products such as surface treatments for carpets and fabrics. It is important to estimate whether PFAS and other contaminants can increase the risk of preeclampsia and other adverse pregnancy outcomes. Mid-pregnancy plasma samples from about 500 randomly selected cases of pre-eclampsia and 500 controls were analyzed. The concentrations of a series of substances were measured. No significant riskincreasing associations to pre-eclampsia were found. An inverse association between pre-eclampsia and the highest quartile of perfluoroundecanoic acid relative to the lowest quartile (relative risk $0.55,95 \% \mathrm{CI}$ : 0.31 0.81) was reported [24]. If this inverse association is replicated by others, the mechanisms behind it should be studied further.

\section{Infection with cytomegalovirus}

Cytomegalovirus (CMV) usually infects early in life, but has a lifelong latent existence in the body. Primary infection or reactivation of the latent virus in pregnancy has been associated with severe outcomes, including fetal death. It is speculated that the infection may interfere with placentation, which can predispose for pre-eclampsia. Mid-pregnancy plasma samples from about 1500 pre-eclamptic women and 1000 controls in MoBa were analyzed with respect to antibody status (IgG and $\operatorname{IgM}$ ). Adjusted for maternal age, parity and smoking, the odds ratio for pre-eclampsia in women positive for CMV $\mathrm{IgG}$ was 0.89 (95\% CI: 0.74-1.05) [25]. In nulliparous women a slight, but statistically significant, protective effect of CMV was found. The results suggest that CMV infection is unlikely to be a major cause of pre-eclampsia.

\section{Validity and selection issues}

The record in the Medical Birth Registry of Norway (MBRN) is included as part of the MoBa database for each pregnancy. All pre-eclampsia projects use this diagnosis as the endpoint. All MoBa pregnancies registered with pre-eclampsia $(n=4081)$ and a randomly selected control group were subjected to further scrutiny. Hospital departments, where the births had taken place, were asked to provide copies of antenatal charts and hospital discharge codes. The antenatal charts include data on presence of increased urinary protein level and blood pressure measurements at the various pregnancy visits. Using this hospital-derived information as the gold standard, the positive predictive value (PPV) of a pre-eclampsia diagnosis in MBRN was found to be $83.9 \%$ [26]. The PPV was higher for primiparous women. This result indicates that there is an element of misclassification of pre-eclampsia in papers that only use the MBRN diagnosis. To the extent that this is non-differential with respect to other variables that are studied, it will tend to weaken the estimated association measures.

In order to estimate the degree of recruitment bias in MoBa, Nilsen et al. [27] estimated prevalences and associations between certain exposures and outcomes in two data sets from the MBRN. One was the MoBa data set and the other was composed of all births in the same period (years 2000 to 2006) in Norway. The prevalence of pre-eclampsia was $3.9 \%$ in MoBa, and $4.0 \%$ in the total population, giving a relative frequency of 0.98 (95\% CI: 0.94-1.01). In addition, the association between pre-eclampsia and parity could be estimated in both data sets. In $\mathrm{MoBa}$, the adjusted odds ratio for pre-eclampsia comparing parity 1 to parity 0 was 0.46 (95\% CI: $0.42-0.50)$, while it was 0.47 (95\% CI: $0.45-0.49)$ in the total population. These figures are reassuring, and suggest that projects using preeclampsia based on MoBa data will most likely not suffer from recruitment bias to any large extent.

\section{CONCLUSIONS AND FUTURE PROSPECTS}

A series of new associations between exposures and pre-eclampsia have now been published using MoBa data. The smoking association was well known, but the novel finding is that it is only smoking in the last trimester that matters. The many associations to nutritional factors and dietary patterns require confirmation. The findings may lead to better advice and guidelines for pregnant women. The new information from studies of abortion and infertility points to biological mechanisms that must be pursued in further research. The strong association between parity and preeclampsia, which is a universal finding, is a mystery that requires more detailed follow-up studies, focusing on immunological mechanisms [28].

Pre-eclampsia is not a new disease. It has almost the same prevalence all over the world. This fact suggests that we are dealing with a disease that is not easily amended by external environmental factors. Preeclampsia is perhaps a consequence of the relentless conflict of interest between the mother and the fetus [29]. The placenta is mainly of fetal origin. Paternally derived genes in fetal cells will provoke maternal responses. The interest of the fetus is to grow as large and mature as possible before birth in order to increase the chance of extra-uterine survival. There is a limit to 
maternal adaptation to this demand, if the mother is to retain her own good health. Thus, there are genes that need to be present for the good health of both organisms. Imprinting is a mechanism that can silence either paternally or maternally derived genes in the growing organism, and should be studied more closely for the development or avoidance of pre-eclampsia. If $\mathrm{MoBa}$ can contribute to finding the genes that are associated with pre-eclampsia, we can close in on the biological pathways leading to the disease. Genetic studies must analyze fetal and maternal genes simultaneously in order to grasp interaction effects. Two large ongoing studies will, in coming years, publish their findings from genome-wide association analyses based on MoBa data. One is the study conducted by Engel as referred to earlier, and the other is an international consortium headed by Linda Morgan, as presented elsewhere in this issue [30].

MoBa's family design means that paternal, fetal and maternal genes can be studied at the same time, opening for studies of maternal-fetal interactions as well as imprinting effects (a certain allele will only have effect when it comes from one of the parents). In addition, many MoBa mothers have participated with more than one pregnancy. Women who have had pre-eclampsia in one but not another pregnancy (discordant pregnancies) are of special interest, since the maternal genome is constant, but the fetal genome and environmental influences differ.

The heterogeneous nature of pre-eclampsia must be better understood. The heterogeneity is partly a phenomenon of degree of severity and partly of timing of events. The current dogma suggests that early pre- eclampsia is related to poor placental blood perfusion due to lack of widening of the maternal spiral arteries. And it is suggested that signals and substances from the compromised placenta will, in predisposed women, lead to the maternal signs of diseases: endothelial damage, increased blood pressure, proteinuria, edema and inflammatory responses in many organs. However, for late onset pre-eclampsia, after 37 weeks of gestation, which constitutes about $60 \%$ of cases, normal placental perfusion is commonly found. Thus, preeclampsia may have two subtypes, and only one of them is associated with poor placentation. Stratified analyses by type of pre-eclampsia should be considered in order to understand the etiology better.

The scientific challenges surrounding preeclampsia are plentiful, and include not only questions on etiology and heterogeneity. We need better insight into pathophysiological mechanisms. The kynurenine finding described above can be helpful in this respect. We also need to find biomarkers and other characteristics during pregnancy that can predict the development of the disease. That will help clinical management. Another line of research is related to the consequences of pre-eclampsia for the mother. There is now a large interest in the association between pre-eclampsia and later cardiovascular disease in the mother. As women with and without pre-eclampsia are followed up, MoBa will assemble data that can shed light on this association. Children exposed to pre-eclampsia in utero should also be followed closely to detect any long-term consequences. MoBa will continue to provide data that will help resolve causes, mechanisms and consequences of pre-eclampsia.

\section{REFERENCES}

1. Lain KY, Roberts JM. Contemporary concepts of the pathogenesis and management of preeclampsia. JAMA 2002;287:3183-6.

2. LeFevre ML, on behalf of the U.S. Preventive Services Task Force. Low-dose aspirin use for the prevention of morbidity and mortality from preeclampsia: U.S. Preventive Services Task Force recommendation statement. Ann Intern Med 2014;161:819-26.

3. Redman CW, Sargent IL. Latest advances in understanding preeclampsia. Science 2005;308:1592-4.

4. Engel SM, Scher E, Wallenstein S, Savitz DA, Alsaker ER, Trogstad L, et al. Maternal active and passive smoking and hypertensive disorders of pregnancy. Risk with trimester-specific exposures. Epidemiology 2013;24:379-86.

5. Meltzer HM, Brantsæter AL, Ydersbond TA, Alexander J, Haugen M. Methodological challenges when monitoring the diet of pregnant women in a large study: experiences from the Norwegian Mother and Child Cohort Study (MoBa). Matern Child Nutr 2008;4:14-27.

6. Brantsæter AL, Haugen M, Myhre R, Sengpiel V, Englund-Ögge L, Nilsen RM, et al. Diet matters, particularly in pregnancy - Results from MoBa studies of maternal diet and pregnancy outcomes. Norsk Epidemiologi 2014;24(1-2): 63-77.

7. Bodnar LM, Catov JM, Simhan HN, Holick MF, Powers RW, Roberts JM. Maternal vitamin D deficiency increases the risk of preeclampsia. J Clin Endocrinol Metab 2007;92:3517-22.

8. Haugen M, Brantsæter AL, Trogstad L, Alexander J, Roth C, Magnus P, et al. Vitamin D supplementation and reduced risk of preeclampsia in nulliparous women. Epidemiology 2009;20:720-6.

9. Hyppönen E, Cavadino A, Williams D, Fraser A, Vereczkey A, Fraser WD, et al. Vitamin D and preeclampsia: original data, systematic review and meta-analysis. Ann Nutr Metab 2013;63:331-40. 
10. FAO/WHO. Guidelines for the evaluation of probiotics in food (page 8). Joint FAO/WHO working group report on drafting guidelines for the evaluation of probiotics in food. London, Ontario, Canada; 2002. Available at: http://www.who.int/foodsafety/fs_management/en/probiotic_guidelines.pdf.

11. Brantsæter AL, Myhre R, Haugen M, Myking S, Sengpiel V, Magnus P, et al. Intake of probiotic food and risk of preeclampsia in primiparous women. The Norwegian Mother and Child Cohort Study. Am J Epidemiol 2011;174:807-15.

12. Clausen T, Slott M, Solvoll K, Drevon CA, Vollset SE, Henriksen T. High intake of energy, sucrose, and polyunsaturated fatty acids is associated with increased risk of preeclampsia. Am J Obstet Gynecol 2001;185:451-8.

13. Borgen I, Aamodt G, Harsem N, Haugen M, Meltzer HM, Brantsæter AL. Maternal sugar consumption and risk of preeclampsia in nulliparous Norwegian women. Eur J Clin Nutr 2012;66:920-5.

14. Brantsæter AL, Haugen M, Samuelsen SO, Torjusen H, Trogstad L, Alexander J, et al. A dietary pattern characterized by high intake of vegetables, fruits, and vegetable oils is associated with reduced risk of preeclampsia in nulliparous pregnant Norwegian women. $J$ Nutr 2009;139:1162-8.

15. Torjusen H, Brantsæter AL, Haugen M, Alexander J, Bakketeig LS, Lieblein G, et al. Reduced risk of preeclampsia with organic vegetable consumption: results from the prospective Norwegian Mother and Child Cohort Study. BMJ Open 2014;4:e006143.

16. Hillesund ER, Øverby NC, Engel SM, Klungsøyr K, Harmon QE, Haugen M, et al. Associations to adherence to the new nordic diet with risk of preeclampsia and preterm delivery in the Norwegian Mother and Child Cohort Study (MoBa). Eur J Epidemiol 2014;29:753-65.

17. Magnus P, Trogstad L, Owe KM, Olsen SF, Nystad W. Recreational physical activity and the risk of preeclampsia: A prospective cohort of Norwegian women. Am J Epidemiol 2008;168:952-7.

18. Haugen M, Brantsæter AL, Winkvist A, Lissner L, Alexander J, Oftedal B, et al. Associations of prepregnancy body mass index and gestational weight with pregnancy outcome and postpartum weight retention: a prospective observational cohort study. BMC Pregnancy Childbirth 2014;14:201.

19. Trogstad L, Magnus P, Skjærven R, Stoltenberg C. Previous abortions and risk of pre-eclampsia. Int $J$ Epidemiol 2008:37:1333-40.

20. Trogstad L, Magnus P, Moffett A, Stoltenberg C. The effect of recurrent miscarriage and infertility on the risk of pre-eclampsia. BJOG 2009;116:108-13.

21. Moffett A, Hiby SE. How does the maternal immune system contribute to the development of pre-eclampsia? Placenta 2007;28 (Suppl A):S51-6.

22. Farrell LE, Hiby SE, Apps R, Chazara O, Trogstad L, Gjessing HK, et al. KIR and HLA-C: Immunogenetic regulation of human birth weight. Norsk Epidemiologi 2014;24(1-2): 107-110.

23. Nilsen RM, Bjørke-Monsen A-L, Midttun Ø, Nygård O, Pedersen ER, Ulvik A, et al. Maternal tryptophan and kynurenine pathway metabolites and risk of preeclampsia. Obstet Gynecol 2012;199:1243-50.

24. Starling AP, Engel SM, Richardson DB, Baird D, Haug LS, Stuebe AM, et al. Perfluoroalkyl substances during pregnancy and validated preeclampsia among nulliparous women in the Norwegian Mother and Child Cohort Study. Am J Epidemiol 2014;179:824-33.

25. Strand KM, Odland ML, Iversen A-C, Nordbø SA, Vik T, Austgulen R. Cytomegalovirus antibody status at 17-18 weeks of gestation and pre-eclampsia: a case-control study of pregnant women in Norway. BJOG 2012;119:1316-23.

26. Klungsøyr K, Harmon QE, Skard LB, Simonsen I, Austvoll ET, Alsaker ER, et al. Validity of pre-eclampsia registration in the Medical Birth Registry of Norway for women participating in the Norwegian Mother and Child Cohort study, 1999-2010. Paediatr Perinatal Epidemiol 2014;28:362-71.

27. Nilsen RM, Vollset SE, Gjessing HK, Skjærven R, Melve KK, Schreuder P, et al. Self-selection and bias in a large prospective pregnancy cohort in Norway. Paediatr Perinatal Epidemiol 2009;23:597-608.

28. Trogstad L, Magnus P, Stoltenberg C. Pre-eclampsia: risk factors and causal models. Best Pract Res Clin Obstet Gynaecol 2011;25:329-42.

29. Haig D. Genetic conflicts in human pregnancy. Quart Rev Biol 1993;68:495-532.

30. Morgan L, McGinnis R, Steinthorsdottir V, Svyatova G, Zakhidova N, Lee WK, et al. InterPregGen: genetic studies of pre-eclampsia in three continents. Norw J Epidemiol This issue 